\title{
Kernos
}

Revue internationale et pluridisciplinaire de religion grecque antique

5 | 1992

Varia

\section{Expérience orgiastique et composition poétique : le Dithyrambe II de Pindare (fr. 70B Snell-Maehler)}

\section{Emilio Suárez de la Torre}

\section{(2) OpenEdition}

\section{Journals}

Édition électronique

URL : http://journals.openedition.org/kernos/1060

DOI : 10.4000/kernos. 1060

ISSN : 2034-7871

Éditeur

Centre international d'étude de la religion grecque antique

Édition imprimée

Date de publication : 1 janvier 1992

ISSN : 0776-3824

Référence électronique

Emilio Suárez de la Torre, « Expérience orgiastique et composition poétique : le Dithyrambe II de Pindare (fr. 70B Snell-Maehler) », Kernos [En ligne], 5 | 1992, mis en ligne le 19 avril 2011, consulté le 21 avril 2019. URL : http://journals.openedition.org/kernos/1060 ; DOI : 10.4000/kernos.1060 
Kernos, 5 (1992), p. 183-207.

\section{EXPÉRIENCE ORGIASTIQUE ET COMPOSITION POÉTTQUE :}

\section{LE DITHYRAMBE II DE PINDARE (FR. 70 B SNELL-MAEHLER)}

1. Malgré la découverte de plusieurs textes sur papyrus depuis la fin du XIXe siècle, on peut affirmer que nous avons encore des difficultés à donner une définition simple et concise du dithyrambe en tant que genre poétique ${ }^{1}$. Une conséquence remarquable de ces découvertes est de nous avoir permis de comprendre la richesse qui se cache sous un terme apparemment simple et qui aurait dû être "univoque». Ceci a soulevé des soupçons à l'égard des classifications anciennes des genres, et pas seulement en ce qui concerne le dithyrambe ${ }^{2}$. D'ailleurs, les nouveaux textes appartiennent à une période très limitée de l'histoire du dithyrambe, la première moitié du Ve siècle av. J.-C. Nous ignorons toujours

1 Pour l'histoire du dithyrambe, cf. W. Schmid, Zur Geschichte des griechischen Dithyrambus, 1901; O. CRUsIUs, in RE, V, col. 1203-1230; G.A. PRIVITERA, Archiloco e il ditirambo letterario presimonideo, in Maia, 9 (1957), p. 95-110; ID., Il ditirambo da canto cultuale a spettacolo musicale, in Cultura $e$ Scuola, 43 (1972), p. 56-66, cf. C. CALAME (éd.), Rito e poesia corale in Grecia. Guida storica e critica, Roma-Bari, 1977, p. 27-37; H. SchöNEWOLF, Der jungattische Dithyrambus, Giessen, 1958; A. PICKARD-CAMBRIDGE, Dithyramb, Tragedy, and Comedy, Oxford, 1962²; R. SEAForD, The Hyporchema of Pratinas, in Maia, 29 (1977-1978), p. 81-94; J.L. MELENA, Perfiles generales para una historia del ditirambo como género literario, in Tabona, n.s. 4 (1983), p. 181-223; M.J.H. VAN DER WEIDEN, The Dithyrambs of Pindar, Amsterdam, 1991, p. 1-33. On attend avec intérêt la publication du Habilitationsschrift du Prof. B. Zimmermann (Zürich), Studien zum griechischen Dithyrambos, Konstanz, 1988. Un recueil des témoignages et une relation des textes peuvent se trouver dans l'œuvre de C. DEL GRANDE, Ditirambografi : Testimonianze e frammenti, Napoli, 1947, ainsi que dans la plus récente de D.F. SuTTON, Dithyrambographi Graeci, Berlin, 1989 (mais attention aux erreurs !). Sur quelques traits du dithyrambe pindarique, cf. R. HaMiLTon, The Pindaric Dithyramb, in HSPh, 93 (1990), p. 211-222.

2 Témoignages dans H. FäRBER, Die Lyrik in der Kunsttheorie der Antike, München, 1936; pour la discussion sur la classification générique, cf. A.E. HARVey, The Classification of Greek Lyric Poetry, in CQ, 49 (1955), p. 157175; L.E. RossI, I generi letterari e le loro leggi scritte e non scritte nelle letterature classiche, in BICS, 18 (1971), p. 69-94; C. CALAME, Réflexions sur les genres litteraires en Grèce archaique, in QUCC, 17 (1974), p. 113-128 et R.L. FOWLER, The Nature of Early Greek Lyric Poetry: Three Preliminary Studies, Toronto, 1987, p. 86 sq. 
comment était le chant qu'Archiloque savait si bien $\dot{\varepsilon} \xi \alpha \dot{\alpha} \rho \chi \varepsilon v^{3}$, ainsi que les traits du dithyrambe simonidéen, pour ne mentionner que deux moments très importants de son histoire.

Néanmoins, sans nous engager dans les points les plus délicats de la polémique autour du dithyrambe, je pense qu'il existe un certain accord général sur les questions suivantes:

a) À partir d'une origine rituelle (et spécifiquement dionysiaque), une variété hymnique (évidemment dédiée à Dionysos) s'est remplie d'un contenu narratif plus ou moins large et d'une certaine complexité structurale qui peut être mise en rapport aussi avec des changements dans les aspects musicaux, métriques, choréographiques, etc.

b) Le fameux «hyporchème» de Pratinas ${ }^{4}$ pourrait être, d'après la définition de Seaford ${ }^{5}$, un exemple de "style dithyrambique pré-pindarique", bien qu'avec des indices de dramatisation et avec des traits de langue qui ne doivent pas être considérés comme définitifs ou exclusifs pour la caractérisation du genre.

c) L'existence d'un type de chorale plus ou moins défini - et qui s'est développé avec une certaine autonomie "générique» dans un contexte agonal dionysiaque précis - n'empêche ni l'emploi parfois «non marqué» du terme - pour décrire un chant choral quelconque de contenu narratif mythique -, ni la confusion - plutôt tardive - avec des éléments du péan ou même avec celui-ci.

Compte tenu de l'état actuel de nos connaissances sur le dithyrambe, il est donc raisonnable, quant à l'état de son évolution au Ve siècle, de se contenter d'encadrer ce genre dans le stade 2 de la division proposée récemment par Fowler ${ }^{6}$ - c'est-à-dire, une poésie aux traits littéraires développés, mais en rapport avec une occasion déterminée - et de nous borner à nous faire une idée de ce qu'était le dithyrambe dans la première moitié du siècle seulement à partir de ce que nous laissent entendre les textes dont nous disposons. En même temps, il faudra établir une comparaison avec le reste des compositions des mêmes auteurs, les renseignements que nous possédons sur les étapes anté-

3 Fr. 120, 2 West.

$4 \quad P M G, 708$.

$5 \quad$ Art. cit., p. 92.

6 Op. cit., p. 90 : «We may distinguish therefore three stages : (1) purely 'occasional' poetry; (2) poetry with developed literary characteristics, but still connected in some way with an occasion; (3) purely bookish or literary genres'». L'auteur admet aussi qu'il y a des exemples qui appartiennent à des stades "liminaux" et que cette classification doit être comprise avec une certaine souplesse. 
rieures et ce que nous savons de l'évolution tardive, si nous voulons arriver à une approche rigoureuse de ce type de composition, sans a priori.

2. Le dithyrambe II de Pindare est un exemple extraordinaire de ce genre de poésie. Je vais aborder son commentaire en l'utilisant comme illustration de l'adaptation parfaite de la composition lyrique à la fête religieuse, c'est-à-dire de la contribution de la technique poétique à l'expérience religieuse d'une telle façon que, sans être une poésie strictement cultuelle et, pour ainsi dire, "de cérémonie», elle devient un élément fondamental dans l'ensemble de la festivité dans laquelle elle s'encadre, et qui synthétise parfaitement les sentiments religieux de la collectivité à laquelle elle est destinée.

La publication, en 1919, par B.P. Grenfell et A.S. Hunt du P. Oxy. 1604 - dans lequel on trouve aussi des fragments appartenant à deux autres dithyrambes ${ }^{7}$ - nous a mis en présence d'un exemple d'édition de ce texte datant du IIe siècle ap. J.-C. qui s'ajoute à d'autres fragments du même poème connus auparavant par transmission indirecte ou même par un autre papyrus de qualité inférieure ${ }^{8}$. Outre ces lignes, on a supposé que, d'après le titre - dans lequel les noms d'Héraclès et de Cerbère ne posent pas de problèmes ${ }^{9}$-, on pourrait assigner au même poème deux autres fragments, les numéros 249 a et 81 de l'édition de

7 Vol. XIII; les autres textes sont le dithyrambe pour les Argiens, édité sous le $n^{\circ}$ I (fr. 70a) par Snell-Maehler, et le dithyrambe pour les Corinthiens ( ${ }^{\circ}$ III, fr. 70c Snell-Maehler). Il faut souligner la grande qualité de cette première édition, avec introduction et commentaire exhaustifs, pleine de remarques brillantes.

8 Les vers 1 et 8-11 étaient déjà conservés chez STRABON, $\mathrm{X}, 3,13$, dont la source est probablement Poséidonius; les vers 1-3 se trouvent chez ATHENEE, XI, 30 et $\mathrm{X}$, 82, et DenYs D'HALICARNASse, Comp. verb., XIV. Les vers 8-18 avaient été transmis - mais dans un assez mauvais état - dans le Pap. Berol., 9571 (v. 44-55), connu comme Anonymus de dithyrambo. Finalement, PluTARQue, Qu. conv., 1, 5, 2 (Mor., 623b); 7, 5, 4 (Mor., 706e), cite les v. 13 et 14. Le papyrus ne manque pas de surprises, tant dans le texte que dans ses scholies.

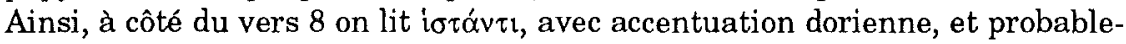
ment comme correction d'une lecture iớ $v \tau_{\imath}$ (sur le corps du texte on lit

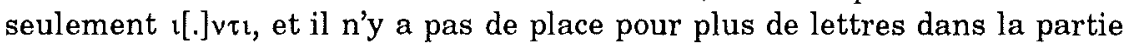
détériorée).

9 Il y a quand même quelques difficultés dans la lecture du premier mot et dans la terminaison du nom du héros. On hésite entre $\Theta P A \Sigma[Y \Sigma]$ HPAKAH $\Sigma$

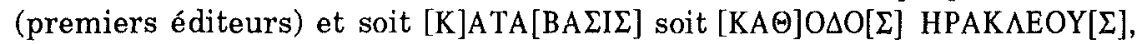
soit $[\Theta \mathrm{H}] / \mathrm{BAIO}[\mathrm{I} \Sigma]$ HPAK $\Lambda \mathrm{H} \Sigma:$ F. FERRARI, Contributi al testo dei Ditirambi di Pindaro, in $S I F C, 9$ (1991), p. 2-8); le reste ne pose pas de problèmes : H

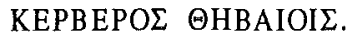


Snell-Maehler. Cette assignation se heurte à quelques difficultés qui ont été déjà soulignées par les premiers éditeurs, mais elles ne sont pas du tout insurmontables ${ }^{10}$.

Ce poème, ainsi que les autres dithyrambes qui l'accompagnent, ont amené les premiers éditeurs à s'exprimer de la façon suivante : «Pindar as dithyrambist was distinctly conservative, and the innovations introduced in the fifth century B.C. were not due to him» ${ }^{11}$. Cette affirmation ne s'approche de la vérité que dans une certaine mesure et elle est un peu hardie si l'on tient compte de notre manque de données sur le dithyrambe précédent. Ce qui est plus surprenant encore est que le poème commence par une déclaration de "nouveauté» par rapport à "l'ancien style». Les commentateurs se sont concentrés sur

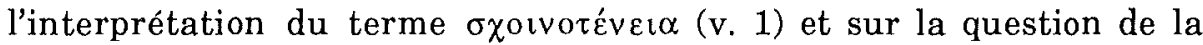
prononciation du san, ce qui les a conduits à des discussions, d'un côté, sur la distribution strophique de ce type de chant, et de l'autre, sur le problème des rapports avec le dithyrambe de Lasse d'Hermione, le maître de Pindare. Mais je pense que la "marque d'identité» du dithyrambe pindarique doit être cherchée à travers l'analyse détaillée de tous ses éléments et que, en ce qui concerne ce dithyrambe II, la phrase qui

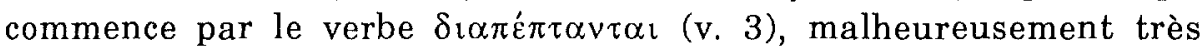
détériorée, était très importante, car elle nous offrait précisément, je pense, le contraste avec l'ancienne modalité dithyrambique à laquelle ce poème s'oppose. Nous reviendrons sur ce point. Je me bornerai, pour

10 Les objections seraient celles-ci : 1) Si le fragment 249b (dans lequel on parle de la is 'A $\chi$ ع $\lambda \omega$ íov) appartient à la même composition que le résumé qui apparaît comme fr. $249 \mathrm{a}$ - avec la mention du thème de Déjanire et l'Achéloos, à propos de la descente d'Héraclès aux enfers et de sa rencontre avec Méléagros -, il faut écarter l'union, car le mètre est différent de celui du dithyrambe. 2) Le fragment 81 présente le même mètre, mais il s'agit ici de paroles adressées à Gérion, qui n'intervient pas dans l'aventure de la descente. Mais, contre ces objections, on pourrait présenter les arguments suivants : 1) Il n'est point nécessaire que le fragment $249 \mathrm{~b}$ appartienne au même poème que le fragment 249a. L'expression citée ci-dessus fait partie d'une énumération

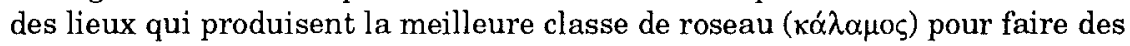
flûtes et elle n'est pas en fonction d'un épisode ou d'un récit tel que celui du fragment $249 a$ - outre le fait de se trouver dans des scholies homériques différentes. 2) Rien ne nous permet d'écarter définitivement le fragment 81 à cause du contenu, puisqu'on pourrait être en présence de l'évocation d'une aventure au cours d'une autre. En plus, la coincidence métrique et le fait que la source (ARISTIDE, II, 70) précise qu'il s'agit d'un passage d'un dithyrambe ('́v $\delta \imath \theta \nu \alpha \dot{\alpha} \beta \beta_{\varphi} \tau \imath v i$ ) sont des arguments, à mon avis, plus forts que celui du contenu.

11 Op. cit., p. 29. 
l'instant, à affirmer que, bien que nous ne soyons pas en présence d'un texte «révolutionnaire» du point de vue littéraire, il s'agit quand même d'une composition avec des particularités très bien définies à l'égard du genre en tant que tel et d'un exemple très intéressant d'adaptation à l'entourage concret de la célébration, à l'environnement religieux.

Parmi les traits les plus remarquables du fragment, par rapport à ce qui était déjà connu par d'autres exemples, et en nous bornant aux exemples qui peuvent avoir une importance plus grande du point de vue de l'expérience religieuse, on peut mettre en relief, d'abord l'emploi du dit schema pindaricum (avec raison) dans un contexte identique à celui du dithyrambe transmis par Denys d'Halicarnasse ${ }^{12}$, qui ne laisse maintenant aucune place pour les doutes, au moins dans les vers 8-9

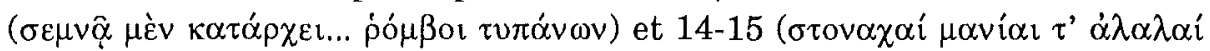

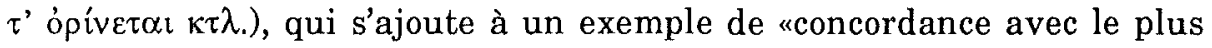
proche» (v. 10-11). Je crois que nous sommes en mesure de parler d'une tendance stylistique à la présentation des moments successifs de la célébration comme une unité, en synthétisant les actions diverses qui ont lieu simultanément.

À côté de ceci, la première strophe nous transmet le deuxième exemple dans la lyrique grecque - dans les poèmes épiques il y a assez de précédents ${ }^{13}$ - d'anaphore de la préposition $\dot{\varepsilon} v$ - employée comme adverbe dans les vers 10,12 et 15 , et en tant que telle avec datif dans le vers 8. L'autre exemple antérieur est celui du célèbre ostrakon florentinum de Sappho (fr. 2). Ce fait a été déjà remarqué par G.A. Privitera, lequel s'exprime sur cette ressemblance dans les termes suivants : "I contenuti caratterizzano diversamente la Stimmung, ma il rapporto del poeta col suo oggetto è identico : è un rapporto di contemplazione» ${ }^{14}$. J'ajouterai qu'il s'agit dans les deux cas de descriptions de lieux qui encadrent l'activité des dieux ${ }^{15}$.

12 Fr. 75 Snell-Maehler. On a proposé l'union de $\tau \varepsilon$ avec les formes verbales dans les vers 18-19, afin d'obtenir un pluriel; cf. MELENA, art. cit., p. 211, avec les références dans la note 114 .

13 Cf. D. FEHLING, Die Wiederholungsfiguren und ihr Gebrauch bei den Griechen vor Gorgias, Berlin, 1969, p. 196.

14 Dioniso in Omero e nella poesia greca arcaica, Roma, 1970, p. 126-127.

15 Il y a, certainement, des différences : le jardin d'Aphrodite est le lieu même de l'expérience religieuse qu'on y décrit (pace C. GaLlavotrit, L'ode saffica dell' ostracon, in Boll. Class., n.s. 28 [1980], p. 3-22, mais cf. G. Pugliese CARRATELLI, Afrodite cretese, in SMEA, 20 [1979], p. 131-141), tandis que l'Olympe reflète tout au plus l'enthousiasme et l'esprit orgiastique de la fête réelle. 
D'ailleurs ce fragment nous offre quelques coïncidences avec des usages pindariques particuliers, déjà remarquées par les premiers éditeurs, mais qui peuvent être complétées. Par exemple, nous trouvons bien attesté chez Pindare l'adjectif $\sigma \varepsilon \mu v \alpha ́$ pour qualifier une déesse : son

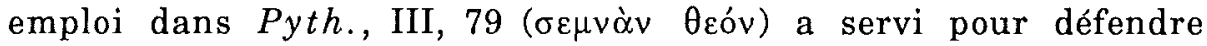
l'identification dans ce dithyrambe de Déméter et de Cybèle ${ }^{16}$. Le

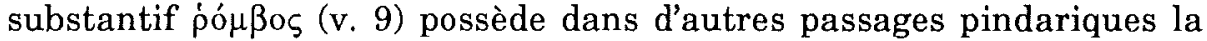
valeur de "mouvement giratoire», "oscillation» 17 , bien qu'on ait pensé aussi à sa forme circulaire pour interpréter l'expression pó $\mu \beta o$ r

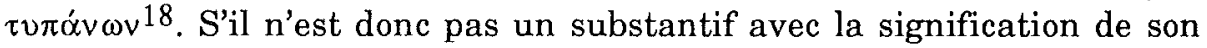
(ce qui est attesté pour d'autres auteurs), on pourrait le considérer comme un phénomène de "synesthésie» issue de l'interaction de la signification du terme et de l'allitération phonique ${ }^{19}$.

Un trait pindarique typique est l'adaptation de la formule épique $\alpha i \theta$ ó $\mu \varepsilon v o v$, qu'on trouve tantôt sans modification $(O l .$, I, 1; gén. Pae., VI, 97), tantôt avec une substitution lexique et disjonction des éléments

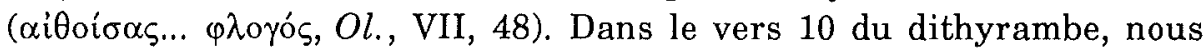

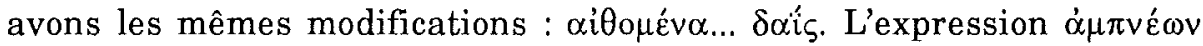
$\pi \hat{\rho} \rho$ (vers 15-16) a aussi son modèle épique, employé seulement pour décrire la Chimère dans $I l$., VI, 182 avec une variation de la formule

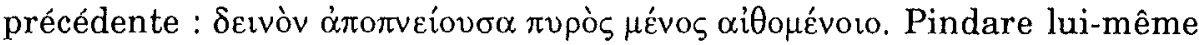
décrit de semblable manière le monstre dans $O l$., XIII, 90 (et cf. $O l$., VII, 71) et les taureaux d'Aiétes dans Pyth., IV, 225, tandis que dans le fr. 146 était attesté déjà un emploi proche de celui que nous avons ici : $\pi \hat{\rho} \rho$ $\pi \vee \varepsilon ́ o v \tau o \zeta . . . \kappa \varepsilon p \alpha v \vee \circ \hat{v}^{20}$.

16 Cf. infra, § 3-4.

17 Cf. Ol., XIII, 93; Isth., IV, 47 : «Waving» traduit par W.J. SLATER, Lexicon to Pindar, s.v.

18 A. Puech traduit : «les timbales rondes ouvrent le ban» (Pindare, tome IV, Isthmiques fragments, Paris, Les Belles Lettres, 1961, p. 148).

19 D'autres emplois lexiques propres de Pindare sont : celui du vers 10, кé $\chi \alpha \alpha \delta[\varepsilon v]$, dit des sons (ici et dans $O l$., IX, 2) et, métaphoriquement, de la manifestation «exultante» (Slater) de la jeunesse (Pyth., IV, 179); la caractéri-

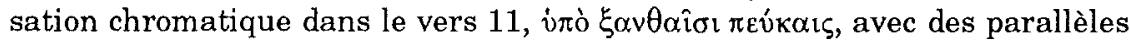

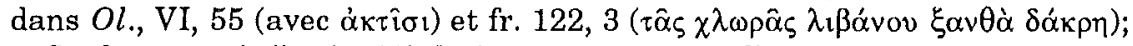
enfin, le terme $\alpha \dot{\gamma} \gamma \hat{\lambda} \alpha \alpha$ (v. 23) désigne un troupeau d'animaux sauvages qui se conduisent comme les domestiques, tout comme dans le fr. 239

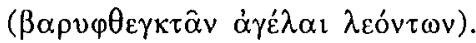

20 L'emplacement initial de l'adverbe $\dot{\rho} i ́ \mu \alpha$ dans le vers 19 rappelle des commencements des vers épiques, tels que celui d'Il., XIII, 30, ’́í $\mu \varphi \alpha \mu \alpha \dot{\alpha} \lambda \alpha$ (dans la description de la vitesse des chevaux de Poséidon); l'association à la 
Le fragment enregistre trois fois la figure (enallagé) qui consiste à qualifier un objet, attribut d'un dieu, avec une épithète de celui-ci. Ainsi,

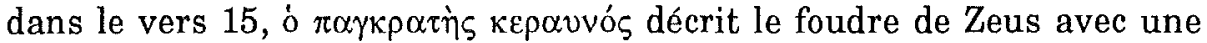
épithète propre au dieu ${ }^{21}$, selon une tendance bien connue chez Pindare ${ }^{22}$. Dans le vers 17 , en reforçant l'allitération, l'égide de Pallas est qualifiée d' $\alpha$ $\lambda \kappa \alpha ́ \varepsilon \sigma \sigma \alpha$, une épithète qui dans l'Hymne homérique, XXVIII, 3 est employée précisément pour Athéna ( $\pi \alpha \rho \theta$ évov $\alpha i \delta o i ́ \eta v$

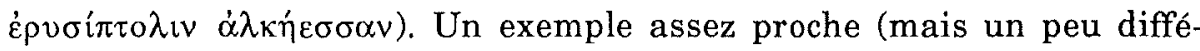

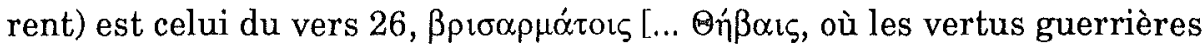
de la cité sont mises en relief par une épithète d'Arès, tel qu'on le trouve dans Hés., Sc., 441 et H.H., VIII, 1.

Ces rapports avec les Hymnes homériques constatés dans l'emploi des épithètes ont une signification spéciale, car il s'agit d'un type de poésie essentiellement religieuse, dont Pindare semble s'approcher. À cet égard je veux remarquer l'importance des similitudes de cette composition avec l'Hymne dédié à la Mère des Dieux (H.H., XIV), dont le texte est le suivant ${ }^{23}$ :

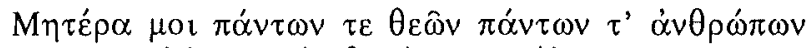

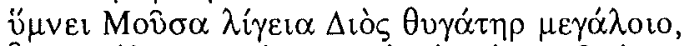

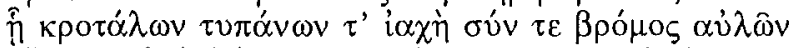

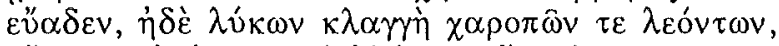

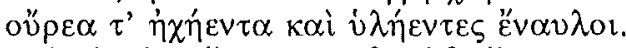

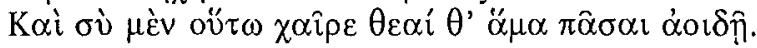

Outre la coïncidence dans la description des instruments (justifiée par le rite), qui créent une ambiance incontestablement orgiastique, on doit remarquer l'usage du substantif $\kappa \lambda \alpha \gamma \gamma$ ' pour la voix des animaux, tel qu'on le trouve dans le vers 18 du fragment (au pluriel) pour les sons (des sifflements ?) produits par les serpents de l'égide Athéna. Cet emploi du substantif apparaît aussi dans H.H., XXVII, 9 (i $\alpha \chi \varepsilon \hat{\imath} \delta^{\prime} \dot{\varepsilon} \pi i$

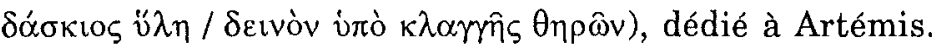

On a donc l'impression que de la même manière que pour les références cultuelles au niveau linguistique, Pindare s'est servi de plusieurs traits de la poésie ureligieuse», assez familiers à l'auditoire,

vitesse des chars se synthétise aussi dans l'expression pindarique $\dot{\imath} \mu \varphi \alpha \dot{\rho} \mu \alpha \tau \sigma \varsigma$

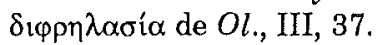

21 Cf. Esch., Th., 255; Eu., 918.

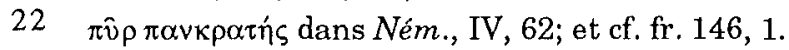

23 Nous suivons l'édition d'Allen-Halliday-Sikes, Oxford, 1936 (réimp. Amsterdam, 1980). 
pour réussir un renouvellement de ces éléments dans le nouveau contexte et pour créer une atmosphère appropriée à la célébration.

Finalement, dans cet exposé sommaire de quelques-uns des procédés formels employés par le poète, il ne faut pas passer sous silence les aspects métriques. Il va de soi que ce fragment ne nous permet pas encore de comprendre pleinement le sens des affirmations d'Horace (seu per audaces nova dithyrambos verba devolvit numerisque fertur lege solutis, Od., IV, 2, 10) ou du Ps.-Censorin (Pindari... qui liberos etiam numeris modos edidit, ch. 9). Néanmoins, l'emploi du vers dactylo-épitrite a attiré souvent l'attention des savants. Wilamowitz ${ }^{24}$ se montrait vraiment surpris par cette façon d'exprimer le délire ('Taumel') dionysiaque avec le mètre qui, à son avis, était le plus contraire à ces sentiments. C'est pour cela qu'il supposait (très justement) que le reste du poème devait avoir un caractère narratif. Pourtant ce fait n'est pas une conditio sine qua non pour le choix métrique; presque aucun des dithyrambes de Bacchylide n'est composé dans ce même mètre ${ }^{25}$. Il faut se demander plutôt si l'emploi de cette versification est significatif d'un point de vue synchronique, c'est-àdire si le choix d'un certain mètre est en rapport avec le contenu et le but de la composition. Il convient de répondre par l'affirmative. Ce choix a une fonction significative, tout en étant un élément stylistique. Il n'y a pas de raison pour ne pas le considérer comme, par exemple, le choix d'un trait dialectal. De ce point de vue - ainsi que par les conséquences que tout cela pourrait avoir pour la danse, que nous ne pouvons pas apprécier - l'emploi du mètre dactylo-épitrite suppose :

a) de doter la composition d'un véhicule apte à un ton épique - qui existe aussi dans la langue - et, évidemment, au caractère narratif déjà remarqué par Wilamowitz;

b) de rapprocher le dithyrambe du genre encomiastique et même de l'épinicie : dans une certaine mesure, ce poème devient une sorte de chant en l'honneur de Thèbes à travers ses traditions mythiques - en commençant par les plus importantes : la légende de sa fondation et la naissance sur son sol du dieu Dionysos ${ }^{26}$.

24 U. von Wilamowitz-Moellendorf, Pindaros, Berlin, 1922, p. 345.

25 Ode, XV, Les Anténorides ou la réclamation d'Hélène, rappelée par GrenfellHunt.

26 Des thèmes et des motifs sont communs aux épinicies et aux autres odes chorales. Dans l'œuvre de C.O. PAvese, La lirica corale greca. I, Alcmane, Simonide, Pindaro, Bacchilide, Roma, 1979, on trouve la relation détaillée pour ce dithyrambe (thèmes : Fe, M3, P3, Praep.; motifs : ant, c, conc, fe, nov, $p r, I I I)$. Même le schéma et les symboles établis par R. HAMilTon, Epinikion. 
3. Le moment est arrivé maintenant d'aborder plus profondément la question de la liaison étroite qui s'établit entre la composition poétique et le contexte cultuel. Si la présence des références religieuses est une particularité constante de la poésie grecque, quand il s'agit d'un chant qui était destiné à une éoptŕ concrète, nous devons nous interroger sur la correspondance des éléments réels du culte et des traditions religieuses locales avec les mentions qu'on y fait de ceux-ci, ainsi que sur leur fonction dans cet ensemble poétique. Plusieurs recherches sur ce sujet se sont avérées extrêmement fructueuses : rappelons ici, à titre d'exemple, l'ouvrage modèle de C. Calame ${ }^{27}$ sur les chœurs féminins en Grèce ou le plus récent d'Eveline Krummen ${ }^{28}$ sur les épinicies de Pindare (qui établit de son côté une ligne de recherche très féconde), ainsi que plusieurs articles de H. Lloyd-Jones ${ }^{29}$ sur les croyances éleusiniennes et orphiques de Pindare ou les travaux de Paola A. Bernardini ${ }^{30}$ sur des poèmes pindariques également (avec des remarques très importantes sur les cultes thébains).

3.1. En ce qui concerne notre fragment, on peut premièrement se demander si cette description si détaillée de la fête dans la demeure des Ouranides comporte des éléments réels. Pour commencer, nous avons ici un procédé déjà connu par d'autres compositions chorales, c'est-àdire la description paradigmatique, sur un plan divin ou mythique lointain, d'une cérémonie identique ou pareille à celle qui se déroule au moment de l'interprétation de l'ode ou qui sert de modèle à une autre fête

General Form of the Odes of Pindar, The Hague, 1974, pourraient être employés pour la description de ce fragment, composé surtout par les motifs (dans sa terminologie) "fonction poétique», "mythe», "louange de la patrie» (avec «exemple mythique») et «invocation poétique».

27 Les chours de jeunes filles en Grèce archaïque (2 vols), Roma, 1977; ID. (éd.), Rito e poesia corale in Grecia. Guida Storica e critica, Roma-Bari, 1977.

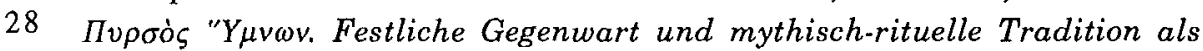
Voraussetzung einer Pindarinterpretation (Isthmie IV, Pythie V, Olympie I und III), Berlin-New York, 1990.

29 Cf. ses articles Heracles at Eleusis : P. Oxy., 2622 and P.S.I., 1931, in Maia, 19 (1967), p. 206-229, et Pindar and the After-Life, in Pindare, VandœuvresGenève, 1985 (Entretiens sur l'Antiquité Classique, 31), p. 245-279.

30 Il proemio della Pitica XI di Pindaro e i culti Tebani, in H. ERBSE - J. BUCKLER (éds), Boiotika. Vorträge vom 5. Internationalen Böotien Kolloquium zu Ehren von Professor Dr. Siegfried Lauffer, München, 1989 [1986], p. 39-47; Iolao in Pindaro : Un Eracle minore?, in A. Schachter (éd.), Essays in the Topography, History and Culture of Boiotia, Montréal, 1990 (Teiresias Supplement, 3), p. 119-123. 
qui est alors évoquée ${ }^{31}$. Dans le cas présent, le lien entre le plan divin et l'actualité du chant s'établit (semble-t-il) à partir de la mention du «nouveau» dithyrambe, en des termes qui font penser à une sorte d'initiation : la métaphore architectonique ( $\delta \iota_{\alpha \pi \varepsilon ́} \pi \tau \alpha \nu \tau \alpha \iota$ est difficile à unir à autre chose que des "portes») ${ }^{32}$, avec des parallèles bien attestés dans d'autres prooimia pindariques, transforme l'ensemble en un lieu sacré ou un temple dans lequel peuvent pénétrer ceux qui ont acquis un 'savoir' que les autres ne possèdent pas. Remarquons que du participe $\varepsilon i \delta o \tau \varepsilon \varsigma$ dépend la phrase interrogative indirecte qui décrit l'orgie divine.

D'emblée il n'est pas suprenant que, destiné à un contexte dionysiaque, le dithyrambe commence par une description de la Bpopíov $\tau \varepsilon \lambda \varepsilon \tau \alpha^{33}$ à laquelle participent plusieurs dieux, car elle est située dans l'Olympe. Le problème est de savoir si cette description est purement conventionnelle ou bien s'il faut compter sur l'influence des éléments de références externes, c'est-à-dire si les dieux et les rites énoncés sont en rapport direct avec la réalité cultuelle ou s'ils ne sont que des ornements littéraires. Une analyse des cultes locaux et d'autres lieux pindariques, ainsi que plusieurs sources, permettent d'affirmer que dans cette description initiale Pindare a fait un amalgame d'éléments majoritairement réels et d'autres (de moindre importance) qui pourraient être classifiés comme conventionnels. On peut affirmer qu'avec ces éléments réels, le poète réussit à décrire une situtation idéale qui, à son tour, possède une fonction paradigmatique pour la fête, le chœur et l'auditoire ${ }^{34}$. Le résultat est, sans doute, assez particulier

31 Par exemple, la fête de noces de Thétis et Pélée dans Ném., V - avec un chœur apollinien!

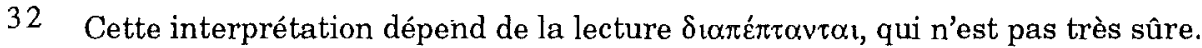
Le dernier éditeur, Van der Weiden, ne rejette pas cette possibilité (op. cit., p. 65), mais, par contre, Ferrari (art. cit., p. 65) propose une forme de

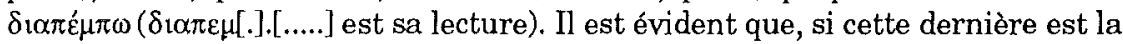
conjecture correcte, mon interprétation n'est plus valable. Mais il faut remarquer que l'interprétation que donne Ferrari n'est pas très satisfaisante : nous aurions ici le motif de «l'envoi» du poème, avec référence «aux nouveaux poètes» (dithyrambographes). Il est difficile d'imaginer chez Pindare une manière aussi maladroite d'introduire ses innovations.

33 Le terme, en singulier ou au pluriel, se trouve dans les autres dithyrambes de Pindare, qui commencent aussi par des références à la célébration dionysiaque.

34 Les éléments qui interviennent ici sont précisément ceux du «rituel ménadique», tel qu'il a été récemment décrit et analysé par J.N. BREMMER, Greek Maenadism reconsidered, in $Z P E, 55$ (1984), p. 267-286, p. 278 sq. 
(Pickard-Cambridge le définissait comme "by far the most striking dithyrambic fragment» ${ }^{35}$ ), mais très efficace du point de vue du 'Erwartungshorizont' religieux et cultuel. À un tel point que quelques savants (O. Schroeder ${ }^{36}$, même Wilamowitz ${ }^{37}$ d'une certaine façon malgré tout ! -, mais surtout H. Fränkel ${ }^{38}$ ), suivant les idées de Nietzsche, n'ont pas hésité à considérer ce fragment comme la matérialisation la plus parfaite de l'esprit dionysiaque, tandis que le prooimion de la Pythique I contiendrait l'essence de l'esprit apollinien, un argument qui a été suffisamment critiqué par Privitera ${ }^{39}$.

3.2. Il va de soi que le «dionysisme» pindarique ne peut pas être considéré d'une façon aussi simple et élémentaire. Surtout, il faut remarquer que, si l'on prend comme point de départ le dithyrambe II, ce n'est pas, et pour cause, un exemple simple. Les particularités du poème ont déjà attiré l'attention de Strabon, qui l'emploie (suivant probablement Posidonius) pour illustrer l'influence et l'extension des rites orgiastiques (à partir de la question des Courètes), dans un passage très connu et souvent commenté. Il conclut que Pindare fait un mélange de

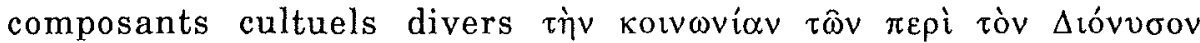

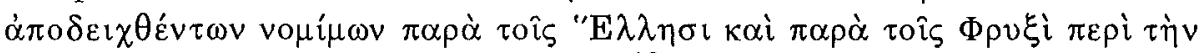

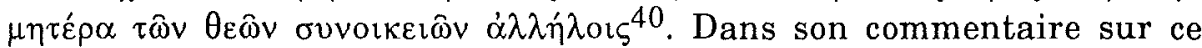
passage l'éditeur des Belles Lettres, F. Lasserre, affirme que, pour Pindare, "le dithyrambe est le chant de fête de Dionysos (Bromios)", tandis que «les instruments de Cybèle n'y participent que dans le concert général des dieux de l'Olympe en son honneur. Posidonius a donc un peu sollicité le texte» ${ }^{41}$. Cela veut dire que l'auteur considère que les éléments orgiastiques appartiennent à une réalité étrangère au rituel dionysiaque. De son côté, A. Pickard-Cambridge propose que le "nouveau" dithyrambe n'ait rien à voir avec le festival céleste de Bromios; en conséquence, cette description aurait un caractère artificiel; cependant il fait remarquer que ce fragment "includes elements which belong, not to the dithyramb but to the trieteric orgies in which the worship and the instruments belonging to the Great Mother

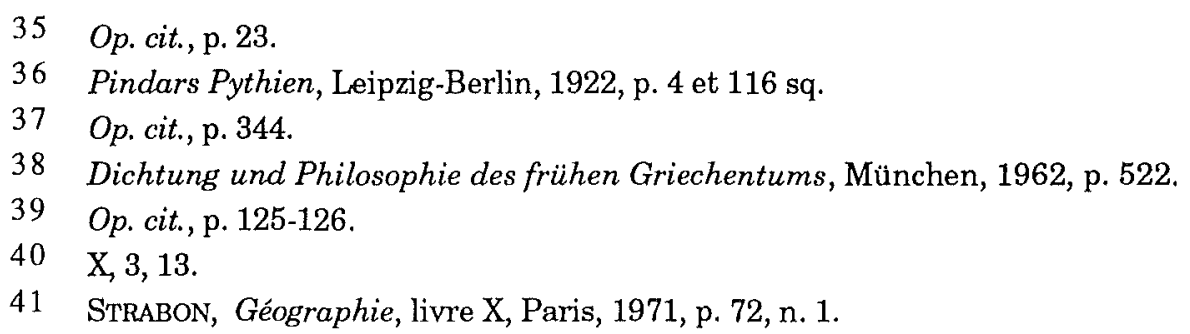


were combined with those of Dionysus" ${ }^{42}$. Mais ici on confirme aussi le vieux principe de quot homines, tot sententiae. H. Jeanmaire ne voyait pas d'inconvénient à considérer le poème comme un exemple des éléments les plus anciens du dithyrambe : "Ainsi le dithyrambe thébain, en dépit du renouvellement que lui vaut la savante poésie qui le stylise, conserve le souvenir que son modèle c'est la ronde démoniaque à laquelle participent en agitant leurs insignes à la manière des thyrses et des nébrides les grands Olympiens dans le déchaînement de l'orchestre de la Grande Mère et des bruyantes acclamations». Il suggérait même que ce fragment nous permettait d'imaginer ce qu'était le dithyrambe d'Archiloque ${ }^{43}$.

3.3. On aperçoit, donc, que ce petit fragment soulève plusieurs questions délicates, surtout en ce qui concerne le niveau chronologique des éléments religieux. Prenons le problème du "dionysisme» de cette composition. D'abord, il faudra admettre que le culte de Dionysos à Thèbes doit être ancien, même s'il est difficile de mesurer son antiquité. La tradition mythique fait de ce dieu, par Sémélé, un petit-fils de Cadmos, le fondateur de Thèbes. Chez Pausanias, et dans deux inscriptions ${ }^{44}$, il est appele Cadméen. Sur la Kadmeia, le nucleus primitif de la ville, il a dû jouir d'un culte ancien. Placer exactement dans le temps ses origines, c'est une question très délicate, que je veux éviter. Les tablettes mycéniennes nous ont transmis le nom du dieu à deux reprises, l'une au nominatif, l'autre au génitif. Cette découverte fut presque révolutionnaire il y a quelques années, car elle obligea à reconsidérer la question de la "nouveauté» de ce culte, telle qu'elle se reflétait dans les traditions grecques. Aujourd'hui nous devons être un peu plus prudents, car l'un des exemples (celui qui est au génitif) est interprété maintenant par les mycénologues comme un simple anthroponyme, et non un théonyme ${ }^{45}$; néanmoins, une nouvelle tablette

42 Op. cit., p. 24.

43 Dionysos. Histoire du culte de Bacchus, Paris, 1951, p. 240.

44 PAUS., IX, 12, 3; I.G., IV, 682; SEG, XIX, 379. Ces inscriptions sont du IIIe siècle

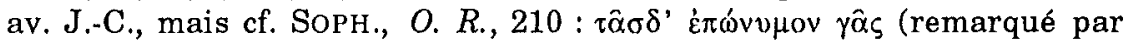
A. SCHACHTER, Cults of Boeotia, vol. I, London, 1981, p. 187, n. 2).

45 Au cours du Colloque Mycénien tenu à Athènes en 1990, E.L. Bennett a démontré que le texte restauré de PY Xa 102 (classé maintenant comme Ea 102), di-wo-nu-so-jo, [ko-to-na GRA qs, doit comporter un anthroponyme au génitif (avec graphie so au lieu de $s i$-jo). Je dois cette remarque à la gentillesse du Prof. M.S. Ruipérez, qui m'a communiqué aussi le texte de son intervention au même Colloque ( À propos de to-so-jo de PY Er 312»), dans laquelle il fait 
semble porter le nom du dieu. Cependant, il existe assez de traces qui font penser à une origine très ancienne du culte dionysiaque, même s'il faut concevoir un 'renouvellement' de quelques aspects du culte, grâce à l'apport des mouvements religieux venus probablement de l'Orient (sans écarter la voie phrygienne ou thrace).

Pourtant, en ce qui concerne Thèbes, et en admettant même qu'un ouvrage comme les Bacchantes d'Euripide ne soit pas inspiré directement, dans tous ses éléments, d'un culte local (A. Schachter ${ }^{46}$, par exemple, pense qu'ils ont été pris des Agrionia et unis à une tradition locale), il n'y a aucune raison pour supposer que cette localisation soit purement fictive : elle a une base réelle. Les agrionia offrent un cadre parfait pour notre fragment 47 .

Et le dithyrambe ? Peut-on penser à un dithyrambe «mycénien»? Je crois que c'est une hypothèse trop hardie et qui crée trop de problèmes ${ }^{48}$. Contentons-nous d'admettre l'existence de traditions qui établissent une liaison entre ce type de chant et des lieux pour lesquels est attestée une continuité archéologique remarquable depuis les périodes mycénienne et sub-mycénienne, soit Naxos, soit Corinthe, soit (last, but not least) Thèbes, toutes les trois connues par Pindare ${ }^{49}$.

Quoi qu'il en soit, je pense que ce fragment nous rapproche encore plus du rite dionysiaque thébain, tel qu'il était probablement pratiqué dans la première moitié du Ve siècle ${ }^{50}$, et qui semble doté de quelques particularités qui font de lui quelque chose de «nouveau», au moins du point de vue des rites locaux. Ce fait a aussi des conséquences quant à, disons, la «religion pindarique». Privitera ${ }^{51}$ a démontré comment Wilamowitz s'était exprimé à tort en jugeant Dionysos comme un dieu «étranger» à la mentalité du poète thébain. Par contre, affirma-t-il,

référence à cette nouvelle interprétation. - Pour d'autres possibilités, cf. VAN DER WEIDEN, op. cit., p. 27.

47 M.P. NILSSON, Griechische Feste von religiöser Bedeutung, Leipzig, 1906 (1925², réimpr. Darmstadt, 1957), p. 272 (avec référence à Rohde) mentionne la possibilité du caractère funéraire des agrionia thébaines. On peut proposer, à titre d'hypothèse, que notre interprétation de la seconde partie du poème pourrait être un appui pour ce trait de fête thébaine. D'ailleurs il ne faut pas oublier que la plupart des données documentaires pour cette fête à Thèbes appartiennent à sa période «agonistique», qui est déjà tardive.

48 Proposée par MELENA, art. cit., p. 190.

49 Cf., Ol., XIII, 19 et fr. 71 (HDT., II, 375, 12. L).

50 La datation de l'ode est ca. 470 av. J.-C.

51 Op. cit., p. 120 sq. 
«del mondo dionisiaco egli ha colto le manifestazioni essenziali e la legge che le governa"52; c'est pour cela que tous les fragments dithyrambiques de Pindare sont épris d'enthousiasme, d'un pathos extatique nouveau par rapport aux épinicies ${ }^{53}$, d'une exaltation pleine de force et de vie, qui est en accord avec l'image extraordinaire du dieu révélée dans d'autres passages pindariques ${ }^{54}$.

Pourtant, et malgré le haut degré d'acceptation qu'éveillent ces opinions, il ne faut pas oublier que cet enthousiasme (au moins dans ce fragment) n'est pas exclusivement dionysiaque : il est aussi métroaque. C'est le moment donc de se tourner vers la question de la Grande Mère.

3.4. La présence de la Magna Mater est vraiment remarquable. Après l'étude de L. Lehnus ${ }^{55}$ sur l'Hymne à Pan de Pindare et après la révision des cultes de Béotie effectuée par Schachter ${ }^{56}$, on ne peut pas douter de l'influence chez Pindare du culte local de la «Mère des dieux». Le passage le plus problématique est celui de Pyth., III, 77-79, où l'on mentionne une $\pi \alpha v v v x$ is de jeunes filles devant la porte de la maison, soit du poète, soit des membres du chœur (sicilien). Sans nous engager maintenant dans la discussion du "je» poétique, nous pouvons adopter la solution (un peu «de compromis») de Schachter : «Sa description de la Meter a pu être influencée sans doute par sa connaissance d'une déesse-mère vénérée à Thèbes» ${ }^{57}$. Ceux qui se sont opposés plus fortement à une interprétation «biographique» de ces données, comme W.J. Slater ${ }^{58}$ ou M. Lefkowitz ${ }^{59}$, ont raison de mettre en cause la validité des affirmations des commentateurs anciens qui créent un cercle vicieux avec son interprétation des textes pindariques; mais une analyse de ces mêmes données, à côté d'autres, nous mène vers une

Op. cit., p. 126.

Op. cit., p. 128.

54 Op. cit., p. 129.

55 L'inno a Pan di Pindaro, Milano, 1979.

56 Op. cit., supra, n. 43.

57 Op. cit., vol. II (London, 1986), p. 138. Il faut tenir compte aussi des remarques faites par LeHNUS, op. cit., p. 15, n. 39, où il se pose des questions sur la possibilité de connaître vraiment le culte de Dionysos à Thèbes; il se demande en même temps "per quale occasione fu composto Dith. $2 \Theta \eta \beta \alpha$ ío Ł̧". J'ai avancé une réponse à propos de Dionysos.

58 Pindar's House, in GRBS, 12 (1971), p. 141-152.

59 Pindar's Lives, in Classica et Iberica. A Festschrift in honor of J.M.-F Marique, Worcester (Mass.), 1975, p. 71-93 et The Lives of the Greek Poets, London, 1981, p. 57-66. 
autre solution. Lehnus a établi un parallèle très intéressant entre le modèle biographique de Pindare et celui d'Archiloque. Dans ces deux cas, le poète est impliqué dans l'introduction d'un certain culte qui soulève une série de réactions d'opposition de la part de ses concitoyens (qui dans l'exemple d'Archiloque, sont punis d'impuissance par leur refus des innovations dionysiaques). La scholie à Pyth., III, $137 \mathrm{~b}$, qui mentionne l'anecdote de l'apparition à Pindare d'une statue de la Mère des Dieux, vision que l'oracle confirme en indiquant qu'on doit ériger un sanctuaire à la déesse, nous parle également de l'établissement de $\tau \varepsilon \lambda \varepsilon \tau \alpha i ́$ en son honneur. Comme l'a souligné Lehnus, loin d'être une "histoire absurde" (Slater), nous serions en présence des indices de

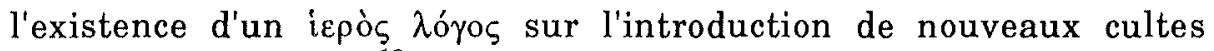
orgiastico-télestiques ${ }^{60}$.

Ce dithyrambe, avec la Pythique III et le fr. 95, confirme l'influence du culte métroaque thébain chez Pindare, dans une modalité qui nous montre la déesse "dans la plénitude de ses attributs asiatiques», d'après Lehnus $^{61}$. Une des particularités locales (qui n'est pas présente dans ce poème) est la proximité de Pan dans sa condition de paredros de la déesse. Les fragments 95-96 de l'Hymne à Pan ne laissent aucun doute. De son côté, Schachter a observé une correspondance concrète avec le culte du Kabirion thébain : les Kabiroi, le père et le fils, y sont les servants de la déesse. L'une de ses manifestations est celle d'Hermès et Pan; pour cet auteur, le complexe cultuel du Cabirion a dû influencer d'une certaine façon les mentions pindariques de la déesse ${ }^{62}$.

Ce fragment a soulevé à nouveau la question de l'identification de Déméter avec la Magna Mater, déjà débattue à propos du passage de la Pythique III. Effectivement, l'argument principal est l'emploi de l'épithète $\sigma \varepsilon \mu v \alpha \dot{\alpha}$ (Pyth., III, $77: \sigma \varepsilon \mu v \dot{\alpha} \theta \varepsilon \varepsilon_{\varsigma}$ ), qui se retrouve ici au vers 8 . Des auteurs tels que B. Moreux ${ }^{63}$, F. Graf ${ }^{64}$ ou A. Henrichs ${ }^{65}$ pensent que, pour Pindare, c'est la même déesse; Schachter est également favo-

60 Op. cit., p. 26.

61 Op. cit., p. 121.

62 Op. cit., p. 93. Contre cette union Cybèle-Pan dans le culte local s'est élevée récemment F. BADER, Autobiographie et héritage dans la langue des dieux: d'Homère à Hésiode et Pindare, in REG, 103 (1990-1992), p. 383-408.

63 B. MoReux, Déméter et Dionysos dans la septième Isthmique de Pindare, in $R E G, 83$ (1970), p. 1-14.

64 F. GRAF, Eleusis un die orphische Dichtung Athens in vorhellenistischer Zeit, Berlin-New York, 1974, p. 155, n. 24.

65 A. HENRICHS, Despoina Kybele : ein Beitrag zur religiösen Namenkunde, in $H S P h, 80$ (1976), p. 253-286 (cf. p. 256-257). 
rable à cette identification et il attire l'attention sur l'épithète de

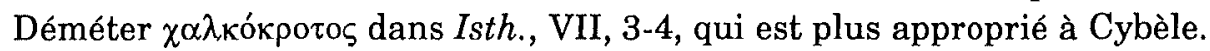
Par contre, Lehnus s'oppose à cette équation (il défend plutôt celle de Rhéa-Cybèle), ainsi qu'à l'influence de l'Attique ou d'Éleusis, supposée par Graf ${ }^{66}$ ou, tout au moins, il ne la considère pas nécessaire ${ }^{67}$.

La question n'est pas facile à trancher (si vraiment il faut le faire $\left.!^{68}\right)$. D'abord nous devons établir une différence essentielle : la Pythique III a été écrite pour un auditoire sicilien. Mais, de l'autre côté, il ne faut pas oublier que, pour Pindare, Dionysos est un paredros de Déméter : il s'exprime ainsi précisément dans les vers déjà cités de l'Isthmique 7, adressée à un Thébain, un fait qui a échappé à G.E. Mylonas quand il a prétendu expliquer l'expression pindarique en ayant recours à la topographie éleusinienne ${ }^{69}$. Quant aux rapports avec Athènes ou Éleusis, je ne saurais pas les écarter définitivement, compte tenu du rapport que j'établirai dans la suite avec un autre fragment douteux de contenu éleusinien.

Pour résumer : il est difficile de savoir si nous sommes en présence d'une identification Rhéa-Cybèle ou Déméter-Cybèle, mais il ne faut pas exclure le commencement de la chaîne postérieure dans cet amalgame, Rhéa-Cybèle-Déméter; considérez ce qui sera après le cas de Phlya, étudié par I. Loucas ${ }^{70}$, avec l'addition de quelques composants orphiques, qui ne sont pas non plus étrangers à notre poète ${ }^{71}$. On peut affirmer que Thèbes semble être le siège, pendant la première moitié du Ve siècle, d'un syncrétisme particulier d'éléments religieux hétéro-

66 Op. cit., p. 151 sq.

67 Op. cit., p. 15, n. 38 .

68 Il faut se demander à quel point l'audience, les fidèles, avaient conscience de ces identifications et si elles étaient significatives du point de vue du culte.

69 Eleusis and the Eleusinian Mysteries, London, 1962 [1961], p. 277-278; cf. p. 278 : "Demeter was established in the Eleusinion in the City below the northwest corner of the Akropolis, Dionysos in the theater below the southeastern corner; thus they became paredroi, that is, they were enthroned near each other".

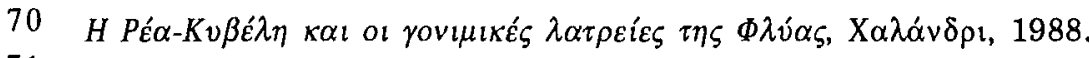

71 Il faut tenir compte qu'une évolution "mystérique» de la Kybaba néo-hittite à la Matar Kubeleya phrygienne avait été proposée par E. LAROCHE, Koubaba, déesse anatolienne, et le problème des origines de Cybèle, in Eléments orientaux dans la religion grecque ancienne, Paris, 1960, p. 113-128 et que cette déesse s'assimile ensuite avec Déméter en Attique; ce qui nous intéresse surtout maintenant est de faire remarquer la présence de ces éléments dans un texte appartenant à une date relativement récente et dans un contexte thébain. 
gènes, dont les voies de pénétration, en ce compris l'Attique, mériteraient une étude détaillée.

3.5. Il convient de mettre en relation avec ce qui précède la présence d'Artémis et la façon de décrire sa participation à la $\tau \varepsilon \lambda \varepsilon \tau \alpha ́$ divine.

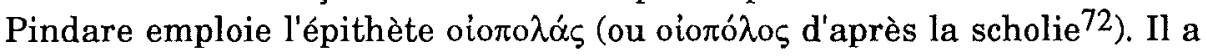
cherché une alternative à celles qui décrivent la déesse comme une

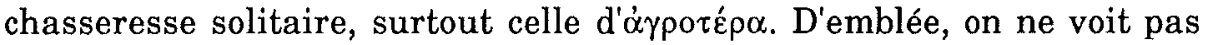
le rapport avec les dénominations thébaines locales de cette déesse, les épiclèses Eileithyia et Eukleia. Mais il n'y a pas de doutes quant à l'existence de son culte thébain, d'après les témoignages de l'EEdipe Roi de Sophocle ou de Pausanias ${ }^{73}$. Un trait remarquable dans ce dithyrambe est l'influence sur les lions (v. 21) qu'il attribue à la déesse. La déesse acquiert de cette façon un caractère "asiatique» proche de celui de Cybèle. On peut penser à un choix purement littéraire de l'auteur (favorisé en l'occurrence par le contexte), mais le fait pourrait aussi avoir assez de valeur, si l'on tient compte de la proximité de cette déesse (Artémis) tant de Déméter que de la Mère des Dieux dans un sanctuaire orgiastique comme celui de Lycosoura, consacré à Despoina ${ }^{74}$, cette Despoina qui, d'après l'hypothèse suggestive d'É. Loucas ${ }^{75}$, devrait être identifiée avec la déesse Artémis, fille, d'après quelques traditions, de Poséidon et de Déméter. Le rôle d'Artémis dans notre fragment est très significatif car il synthétise son ancien caractère de חó $v \imath \_\theta \eta \rho \hat{v} v$ et les traits propres au rite orgiastique et métroaque ${ }^{76}$.

72 Cf. Pyth., IV, 28 avec $\delta \alpha i ́ \mu \omega v$, en parlant d'Eurypyle.

73 Dans l'Edipe Roi apparaissent mentionnées deux fois les triades locales : Athéna-Artémis-Apollon, v. 158-163, et Apollon-Artémis-Dionysos, v. 206-207; cf. aussi PAUS., IX, 11, 4 et cf. SCHACHTER, op. cit, vol. I, p. 104.

74 Des représentations d'Artémis avec des lions sont bien connues à date ancienne, cf. $L I M C$, vol. II, 2 (1984), fig. 2, 21, 33 (a), 3440 c, 51, 64, etc. dans une iconographie qui s'identifie à celle de la Mère des Dieux asiatique.

75 Le nom de la Thea Despoina (TATIEN, Ad Graec., 29 - PAUS., VIII, 37, 6 et 9), in

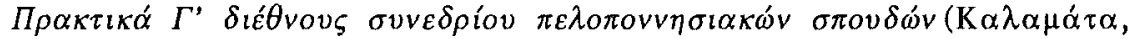
1985), Athènes, 1987-1988, p. 401-419.

76 M.N. Van LoON, Anatolia in the Earlier First Millennium B.C., Leiden, 1990 (Iconography of Religions, XV, 13) apporte des matériaux très intéressants, qui peuvent servir d'appui et d'illustration aux idées que je viens d'exposer, aussi bien en ce qui concerne Déméter (cf. p. 10) qu'à propos d'Artémis (cf. surtout p. 34 pour les rapports entre Artemis - par exemple à Éphèse - et la Mater Kubeleya - Cybèle-phrygienne). 
Par contre, je pense qu'il ne faut pas tenir compte de l'intégration de la déesse dans le culte du Cabyrion (comme c'est le cas pour Délos ${ }^{77}$ ) sur la seule base dans cette enceinte d'une inscription sur un bloc de pierre

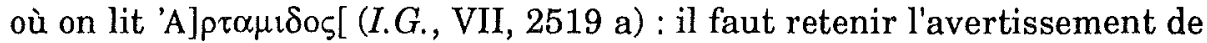
Schachter ${ }^{78}$ selon lequel cette pierre a pu être transportée d'un autre lieu à une date récente (Ier siècle).

3.6. Bien que je ne cherche pas à trouver une correspondance totale entre les divinités mentionnées et les cultes thébains (comme si la composition poétique était un "guide» pour les fidèles), on doit admettre que toutes les divinités qui y interviennent composent un panthéon tout à fait significatif pour les citoyens de la ville cadméenne, bien qu'on ne puisse pas placer au même niveau leur importance dans ce contexte et malgré quelques difficultés pour les localiser dans un cadre local. Si le foudre de Zeus n'offre pas de doutes quant au sens de sa présence à Thèbes (et encore moins dans une composition dédiée à Dionysos, le fils de Sémélé ${ }^{79}$ ), Enyalios ne connaît pas de culte de ce nom à Thèbes et il n'a aucun lien avec les traditions mythiques de la cité, à moins qu'on admette une identification avec Arès, ce qui n'est pas du tout impossible, mais qui pose également un problème un peu délicat. Comme l'avait déjà signalé F. Vian à propos du mythe des Spartes, "son culte n'est attesté à Thèbes que par la scholie à Eschyle, Sept., 103 [sic, mais c'est 105 a] où le commentateur ne semble pas avoir d'autre source que le texte même d'Eschyle» 80 . Mais il hésite avec raison «à sacrifier Arès», étant donné son importance dans le complexe mythique thébain (le dragon était né du dieu et de l'Erinys Tilphossa), ce qui l'amène à proposer (à partir d'une représentation figurée sur une lékané béotienne du Louvre) que «l'Arès thébain pourrait être une ancienne divinité guerrière et chthonienne, parèdre ou époux de la Terre» ${ }^{81}$. Quoi qu'il en soit, son rôle dans le mythe des origines du peuple thébain suffirait à justifier

77 Cf. B. Hemberg, Die Kabiren, Uppsala, 1950, p. 150, sur la présence de la déesse et d'Hécate dans le sanctuaire délien.

78 Op. cit., p. 95, n. 2.

79 I y a une tradition selon laquelle la Cadmée, l'acropole de Thèbes, reçoit aussi

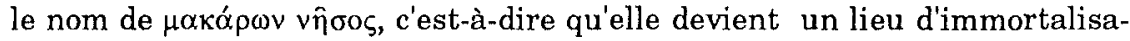
tion depuis que Sémélé fut foudroyée là-bas; cf. W. BurkerT, Elysion, in Glotta, 39 (1961), p. 208-213 (cf. p. 212, n. 2) et G. NAGY, Greek Mythology and Poetics, Ithaca-London, 1990, p. 141. Les sources sont PARMENIDE, dans la

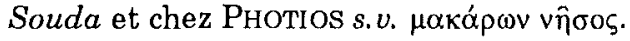

80 Les origines de Thèbes. Cadmos et les Spartes, Paris, 1963, p. 108 et cf. en général le ch. 4, «le dragon et la source d'Arès», p. 94 sq.

81 Op. cit., p. 109. 
son évocation et sa participation dans la fête des dieux décrite dans le poème. Sous cette lumière, l'application au nom de la ville d'une

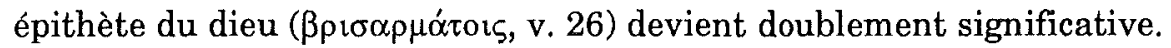

Quant à Athéna, nous sommes dans une position plus favorable. On connaît bien son culte dans la ville comme Onka et, très probablement, Pronaia $^{82}$, et, comme dans le cas d'Arès, elle joue un rôle remarquable dans la légende de la fondation de Thèbes : dans la version d'Apollodore $^{83}$, par exemple, c'est pour elle que Cadmos veut immoler la vache qui l'a conduit jusqu'à l'emplacement définitif; c'est la déesse aussi qui lui conseille de semer les dents du dragon et elle aussi qui, après sa servitude à Arès, lui confềre la royautée 84 .

Enfin, le rôle des Naïades (v. 12) est parfaitement en accord avec le contexte dionysiaque et cohérent avec un autre passage pindarique. Dans le fragment $156^{85}$, la Naïade est le couple de Silénos, le «précepteur» du dieu, décrit par Pindare en des termes aussi très «dionysiaques» :

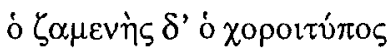

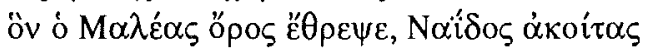

$\sum \imath \lambda \eta v o ́ s$.

Il n'y a rien d'étonnant donc dans le fait que, au moment de chercher un contrepoint "divin" aux chœurs bacchiques qui participent au réel, Pindare ait choisi cette figure. Peut-être que nous ne devons pas voir ici une correspondance exacte avec les jeunes filles de la Pythique III (critiquée par Slater et Lehnus ${ }^{86}$ ), mais très probablement le contexte d'une fête nocturne dionysiaque 87 (avec des éléments orgiastiques inconnus, par exemple, à Athènes $)^{88}$ dans laquelle la participation

82 Cf. Soph., O. R., 20-21, avec les remarques de SChACHTER, op. cit., vol. I, p. 132.

83 III, 4, 1 sq.

84 Cf. VIAN, op. cit. Sur les motifs de cet oracle (avec un exposé des différentes versions) cf. aussi son ch. III, L'oracle et l'animal guide, op. cit., p. 76 sq.

85 Cité par PAUS., III, 25, 2.

86 Celui-ci parle même de 'fantasmagorie' (op. cit., p. 10).

87 C'est la classification de KRUMMEN, op. cit., p. 276 (Dionysisches Nachtfest für Thebaner).

88 Déjà E.R. Dodds, dans son commentaire au vers 59 des Bacchantes (s.v. $\tau \hat{u} \mu \pi \alpha v \alpha$ ), avait fait des remarques dans ce sens concernant les instruments musicaux : «It is, with the flute, the characteristic instrument of the orgiastic 
féminine a pu être fondamentale et grâce à laquelle le dieu se réjouit

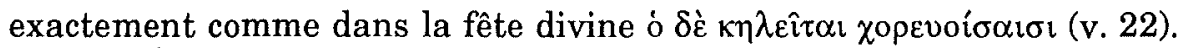

3.7. À la fin de notre fragment, en fonction de la liaison entre le prooimion et la partie narrative sur Héraclès et Cerbère, nous avons un motif qui nous est bien connu par les odes de Pindare : la présence du poète par la bouche du chœur (la «fonction poétique» de R. Hamilton ${ }^{89}$ ), qui se proclame héraut (désigné par la Muse) qui annonce (ou qui prie pour $)^{90}$ la prospérité de Thèbes devant toute la Grèce. La «fonction poétique» est donc unie à un autre motif de caractère encomiastique, la louange de la ville (l'équivalent de la patrie du vainqueur dans les

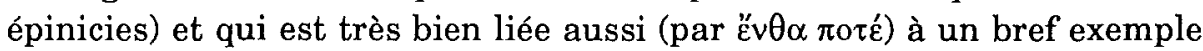
mythique très important : le souvenir du mariage de Cadmos et d'Harmonie, laquelle "écouta la voix de Zeus»91.

Je pense que tout ce passage a une signification assez remarquable, et non seulement en raison de sa cohérence quant à l'emploi du mythe local par excellence (qui nous dévoile ce qui avait été seulement suggéré par les mentions des trois dieux qui interviennent dans la légende). Encore une fois Privitera a avancé des idées importantes sur ce point. Il a signalé que le rapport entre Pindare et le dithyrambe ressemble à celui qui existe entre Dionysos et les choses, c'est-à-dire qu'il provoque dans le chant l'enthousiasme dionysiaque sans être pour sa part envahi par celui-ci. Dans cette ode, le poète regarde et interprète la fête «per grazia delle Muse che hanno destinato lui ad annunziarla»92. Mais il faut remarquer que cela se fait sans renoncer à l'esprit delphique qui illumine incontestablement ses œuvres, non seulement parce que Dionysos est aussi dans une certaine mesure "delphique", mais aussi parce que, de la même façon que la $\varphi \alpha ́ \alpha \alpha$ du peuple conserve le souvenir de diverses manifestations de la voix de Zeus, de sa ó $\mu \varphi \dot{\alpha}$ (oracle à Cadmos ${ }^{93}$, révélation d'Harmonie), le poète, grâce à la Muse, devient encore une fois un interprète de la Muse et des dieux.

cults (...). The tympanum seems to have had no place in the official Athenian worship of Dionysos, which was not orgiastic".

90 Le sens du participe cưxó chez les premiers éditeurs, $a d$. loc.

91 V. 29; VIAN, op. cit., p. 25, n. 4, souligne l'antiquité de cette version chez Pindare par rapport à d'autres auteurs.

92 Op. cit., p. 127.

93 Par l'intermédiaire d'Apollon, évidemment. 
Mais cette fois, il n'est ni $\pi \rho \circ \varphi \eta \dot{\tau} \eta \varsigma^{94}$ ni un $\mu \alpha^{\prime} v \tau \iota \varsigma^{95}$. Il est un $\kappa \eta \dot{\rho} \nu \xi$. Le choix de ce terme possède-t-il une valeur spéciale ici ? Question difficile à résoudre, certainement. Je vais avancer une hypothèse qui peut sembler un peu hardie, mais qui n'est pas, je pense, exagérée. Pendant le Ve siècle, ce titre désignait un office important dans la vie publique des citoyens, mais pas seulement dans les activités profanes. Il y avait également un $\kappa \dot{\rho} v \xi$ très important dans la vie religieuse grecque : le

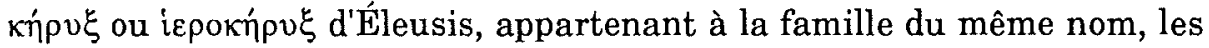
Kérykes. Cependant, pour établir ce lien d'une façon justifiée, il faudrait trouver un argument pour appuyer l'orientation mystique que cette évocation (et cette équivalence entre poète et mystagogue) pouvait susciter dans l'auditoire. La réponse, à mon avis, était dans la partie perdue du poème, car dans celle-ci se racontait, ne l'oublions pas, la descente aux Enfers d'Héraclès.

4. Évidemment la présence du héros thébain par excellence se justifie d'elle-même et nous pourrions considérer suffisantes ces raisons de caractère local pour expliquer le choix du mythe ${ }^{96}$. Cependant je pense que la sélection de cette aventure se fonde sur d'autres éléments. Pendant sa descente, le fils de Zeus luttait contre le terrible chien Cerbère, qui chez Pindare avait, semble-t-il, cent têtes ${ }^{97}$, et rencontrait Méléagros, thème central aussi de l'épinicie 5 de Bacchylide (pour Hiéron de Syracuse), dont le mythe possédait une valeur paradigmatique qui a été très bien analysée par A.P. Burnett ${ }^{98}$. Ce qui m'intéresse maintenant c'est de mettre en rapport ce fragment avec d'autres textes dans lesquels la katabasis d'Héraclès a été employée pour donner au récit une orientation mystérique : plus concrètement, comme aition dans le rituel et les traditions des mystères éleusiniens ${ }^{99}$. N. Robertson

$94 P$ P., VI, 6 et cf. fr. 150. Sur les aspects «prophétiques» de la poésie pindarique cf. mon article Parole de poète, parole de prophète : les oracles et la mantique chez Pindare, in Kernos, 3 (1990), p. 347-358.

95 Fr. 75, 13 (=Dith. 3) et Parth., I, 5.

96 Sur le culte local d'Héraclès, cf. ScHACHTER, op. cit., vol. II, p. 14-30.

97 Fr. 249 b. Cette mention de Cerbère a servi même à proposer l'influence de ce poème chez Horace $(O d ., \mathrm{I}, 37)$, à partir d'une (hypothétique) interprétation tardive du sens général comme «exemplification du tyran renversé" (mort de Cléopâtre) et commémoration du triplex triumphus d'Octave (A. HARDIE, in Horaces Odes 1, 37 and Pindar Dithyramb 2, Papers of the Liverpool Latin Seminar (éd. par F. Cairns), Liverpool, (1976) 1977, p. 113-140.

98 The Art of Bacchylides, Cambridge (Mass.)-London, 1985, p. 129-149.

99 On pourrait croire en contradiction avec ce que je propose les opinions de Mylonas (op. cit., p. 269) dans le sens que : a) Dans les dromena les «enfers et 
a publié un article important sur ce thème $\mathrm{e}^{100}$, dans lequel il poursuit les traces du poème qui, pour ainsi dire, inaugure la série des traitements de ce mythe. Son hypothèse est qu'il s'agit d'un poème épique antérieur à 600 av. J.-C. et, plus concrètement, l'Égimios : le héros réalisait sa katabasis, comme nous le savons par d'autres sources ${ }^{101}$, après avoir été initié aux mystères par Eumolpos. D'après la datation, il faut penser à une période pendant laquelle Éleusis était sous la domination de Mégare, plutôt que celle d'Athènes. Ce poème serait la source originelle des récits et des traitements postérieurs, dont Apollodore nous offre le résumé le plus complet.

Robertson (et c'est le plus remarquable pour nous) attribue une grande importance au témoignage d'un fragment lyrique sur papyrus publié ${ }^{102}$ en 1967 et qui est édité maintenant parmi les fragmenta dubia de Pindare (fr. 346). Très probablement on mentionne dans ce texte l'institution des mystères à Éleusis : Héraclès (et voici la valeur d'aition) interviendrait ici comme le premier initié non éleusinien, un motif qui, d'après Robertson, se trouverait déjà dans le poème épique archaïque. Peut-être la question du précédent épique soulève-t-elle quelques doutes, mais le plus important pour nous maintenant ce sont les particularités du fr. 346 et ses rapports avec notre dithyrambe. Malgré son état, nous pouvons remarquer deux traits qui le rapprochent de ce fragment : l'emploi des dactylo-épitrites et la référence à la rencontre d'Héraclès avec Méléagros pendant la katabasis du premier. Appartenait-il à ce poème ? Du point de vue métrique, les différences dans les kola ne sont pas un obstacle, car ce nouveau fragment pouvait appartenir à l'une des épodes, comme l'a signalé déjà le premier éditeur ${ }^{103}$. L'identification avec le texte pindarique auquel on fait

leurs visions ne jouaient aucun rôle; ceci aurait été une orientation et une interprétation orphiques; et b) il n'existe pas de relation avec Dionysos. Pourtant il faut remarquer que : a) Les croyances orphiques chez Pindare semblent aujourd'hui hors de doute; b) Les Grenouilles d'Aristophane montrent que tous ces éléments (présence de Dionysos, descentes aux enfers, etc.) exerçaient une influence sur l'auditoire; c) Pindare est un «dynamisateur" de la vie religieuse, il peut combiner des éléments, surtout parce que d) ce n'est pas un "guide" d'un culte. Le poète a la liberté de provoquer cette synthèse.

100 Heracles Catabasis, in Hermes, 108 (1980), p. 274-300.

101 Cf. APOLLOD., II, 5, 12.

102 P. Oxy., 2622, vol. XXXII (1967), p. 63-65, qui doit être complété par le P.S.I. 1391, qui contient un commentaire très important du passage.

103 P. 63. 
référence dans la scholie à l'Il., XXI, 194 (c'est-à-dire, au fragment $70 \mathrm{~b}$ Sn.-M.) a été défendue par H. Lloyd-Jones ${ }^{104}$. Les problèmes concernant tous ces textes sont les suivants :

a) D'abord, il faudrait confirmer que le fragment appartenait à un poème de Pindare. Cependant le témoignage de la scholie, d'un côté, et des arguments de langue, mètre, etc. ainsi que des difficultés pour l'assigner à un autre auteur, amènent à le reconnaître comme pindarique.

b) Il faudrait pouvoir démontrer ensuite que ce texte faisait partie du dithyrambe II. La question la plus délicate a été posée par les références éleusiniennes si concrètes du fragment 346, difficiles à harmoniser avec un contexte thébain 105 .

Remarquez, néanmoins, que l'assignation de ce fragment au dithyrambe II n'introduit qu'une différence, disons, de degré. Elle n'est pas une conditio sine qua non pour mon interprétation. Quoi qu'il en soit, il faut reconnaître que la théorie de Graf sur l'influence des courants religieux de l'Attique dans la fusion éventuelle de l'élément éleusinien et la religion métroaque perceptible dans des textes comme l'Isthmique VII, hypothèse à laquelle s'est opposé Lehnus ${ }^{106}$, devrait être admise, au moins quant à la présence indubitable de ces éléments chez Pindare, que le dithyrambe II, à mon avis, reflète sans doute.

Si nous acceptons que le fr. 346 est pindarique (qu'il appartienne ou non au dithyrambe pour les Thébains), nous y aurions la démonstration que, pour ce poète, l'évocation de la katabasis d'Héraclès est en rapport avec des rituels mystériques. Et, si le fragment 346 n'appartient pas au dithyrambe II, nous serions donc en présence d'un exemple "de répertoire" à échelle réduite, qui pourrait être adapté à un auditoire concret.

À mon avis, tout cela favorise de façon convaincante le rôle du poète dans la diffusion des doctrines et des courants religieux de son temps: l'orphisme (généralement admis déjà, après les études de J. Duchemin ${ }^{107}$, H. Lloyd Jones ${ }^{108}$ ou F.J. Nisetich ${ }^{109}$ ), exprimé à

\footnotetext{
104 Art. cit. supra, n. 29, «Heracles at Eleusis...».

105 L'autre référence importante aux mystères d'Éleusis chez Pindare se trouvait dans un poème pour un Athénien, Hippocrate (fr. 137), en des termes qui ne laissaient pas beaucoup de place pour le doute quant à son caractère d'expérience mystique.

106 Cf. supra, p. 197.

107 Pindare, poète et prophète, Paris, 1955.

108 Art. cit. supra, n. 29.
} 
travers une tradition poétique enrichie par lui-même; le culte de la Grande Mère, le dionysisme, les mystères éleusiniens. Et tout cela chez un poète dont l'esprit delphique était incontesté ! Et il ne faut pas oublier que la manifestation de toutes ces croyances religieuses dans les différentes compositions ne peut pas être séparée des particularités strictement poétiques, de la recréation constante du langage et des structures.

5. Ce dithyrambe, comme je l'avais avancé, est un exemple de contribıtion de la technique poétique à l'expérience religieuse, un phénomène qui dépasse les limites et les conditionnements stricts du genre. Pour les vérifier, il faut simplement additionner tous ces éléments que nous avons distingués jusqu'à présent et réfléchir sur leur disposition et sur leurs effets possibles sur un auditoire déterminé. Il y a une proclamation initiale de contraste avec ce qui était auparavant le chant des dithyrambes. Je pense que ce qu'implique le terme $\sigma \chi 0 เ v o \tau \varepsilon ́ v \varepsilon ı \alpha$ vise au-delà de la différence entre astrophique et strophique (par exemple, veut-il se détacher du «dithyrambe» purement narratif et privé d'éléments, pour ainsi dire, religieux ?) et que le regret de "falsification" qu'enferme l'adjectif $\kappa i ß \delta \eta \lambda$ ov dépasse les limites purement phoniques. Il y a surtout un grand effort d'adaptation du chant aux exigences d'authenticité de l'expérience religieuse concrète. De la même façon que dans le dithyrambe aux Athéniens dont nous avions connaissance par Denys d'Halicarnasse ${ }^{110}$, on trouve une invitation aux dieux à venir sur l'agora d'Athènes; le prooimion que nous venons d'étudier fait s'approcher les plans divin et humain, avec la fusion des éléments réels de la fête célébrée et de celle des Olympiens. Le chant ouvre ses portes à une participation qu'amène l'audience de la fête des dieux aux portes de l'Hadès. Le rite orgiastique ajoute à sa frénésie, à travers le chant, l'expérience épouvantable et purificatrice en même temps, de la descente aux Enfers, dans laquelle Héraclès a reçu la révélation de son destin immortel.

Privitera avait ouvert une bonne voie d'interprétation de ce dithyrambe quand il souligna que la «nouveauté» de Pindare n'était que la restauration $d u$ dithyrambe dionysiaque authentique. Cette analyse permet de considérer que cette rénovation du chant en l'honneur de Dionysos impliquait aussi le fait de le doter des moyens de s'adapter à

109 Immortality in Acragas. Poetry and Religion in Pindar's Second Olympian Ode, in CPh, 83 (1988), p. 1-19; Pindar and Homer, Baltimore-London, 1989, passim.

110 Fr. 75, pour les Athéniens; cf. les remarques d'A. MotTE, Prairies et jardins de la Grèce antique, Bruxelles, 1973, p. 39. 
des exigences religieuses très complexes, nées de riches courants existant en ce temps-là dans toute la Grèce et, en l'occurrence, à Thèbes.

Departemento de Filología Clásica

Emilio SUÁREZ DE LA TORRE

Facultad de Filosofía y Letras

Universidad de Valladolid

E - 47002 VALLADOLID 